\title{
Familial restrictive cardiomyopathy with atrioventricular block and skeletal myopathy
}

\author{
A P Fitzpatrick, L M Shapiro, A F Rickards, P A Poole-Wilson
}

\section{Abstract}

Five generations of an Italian family with an autosomal dominant restrictive cardiomyopathy are described. Members of four generations were examined. Symptoms usually developed in the third or fourth decade but the disease did occur in childhood. Initially the condition was characterised by normal ventricular size and systolic function with increased diastolic filling pressures in both ventricles and consequent bi-atrial enlargement. Cardiac catheterisation showed a left ventricular filling pattern of "dip and plateau". The electrocardiogram typically showed non-specific changes in the ST segment and $T$ wave and changes indicating considerable atrial enlargement, which were confirmed by echocardiography. Light microscopy of two endocardial biopsy specimens showed no specific features but excluded the endomyocardial fibrosis of eosinophilic heart disease, amyloid, and specific heart muscle diseases. At necropsy in one case examined under light microscopy extensive patchy fibrosis was found throughout the endocardium, myocardium, and subepicardium, but there were no features typical of eosinophilic heart disease. Histopathological and biochemical examination of skeletal muscle identified no abnormality. The disease often had an insidious course over five to ten years after presentation. Bundle branch blocks, leading to complete atrioventricular block, however, often occurred and may be the first manifestation. Some individuals who survived into the fifth decade developed a progressive, nonwasting skeletal myopathy.

Restrictive cardiomyopathy gives rise to impaired compliance, impedance to ventricular diastolic filling, and cardiac failure. The best recognised cause of this abnormality is the spectrum of diseases encompassing Davies's endomyocardial fibrosis and Loffler's endocarditis. ${ }^{1}$ Typically, in this restrictive cardiomyopathy there is gross endocardial fibrosis. This represents the end stage of a process that starts with inflammation, with eosinophilic infiltration, and myocardial necrosis. $^{2}$ Frequently there is endocardial thrombus formation, leading to thromboembolism. There may be involvement of the atrioventricular valves and cavity obliteration. Other specific heart muscle disorders such as amyloid and haemochromatosis can also produce the haemodynamic characteristics of myocardial restriction.

We describe an hereditary disease producing features typical of ventricular restriction but without the gross endocardial changes usually seen. There was an associated but uncharacterised, non-wasting skeletal myopathy. The aetiology and pathogenesis are unknown.

\section{Patients and methods}

CLINICAL DATA

Two siblings (cases III.9 and III.10) received cardiac pacing for atrioventricular block. There was limited investigation at the time of pacing.
National Heart Hospital, London A P Fitzpatrick L M Shapiro A F Rickards A F Rickards
P A Poole-Wilson

Correspondence to Professor P A Poole-Wilson, National Heart and Lung Institute, Dovehouse Street, London SW3 6LY.

Accepted for publication 21 September 1989

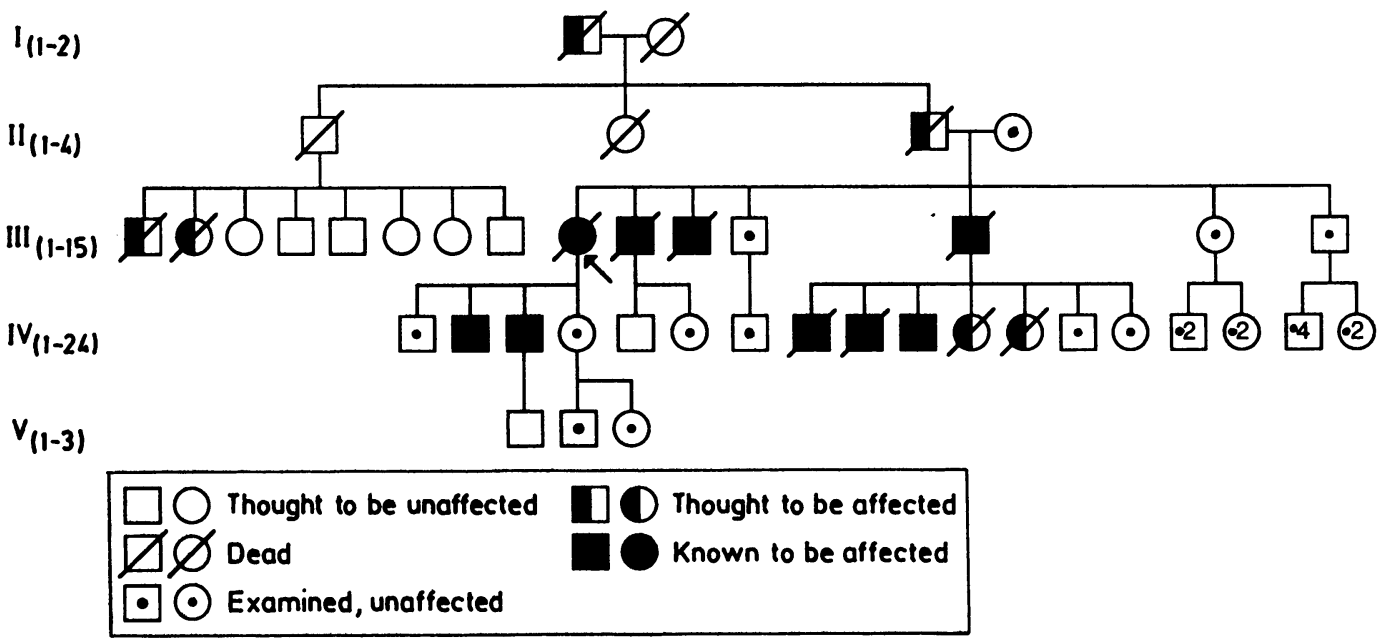

Figure 1 Family pedigree. 
Figure 2 Left ventricular pressure trace in case III.10 showing a typical "dip and plateau" diastolic waveform of restrictive disease.

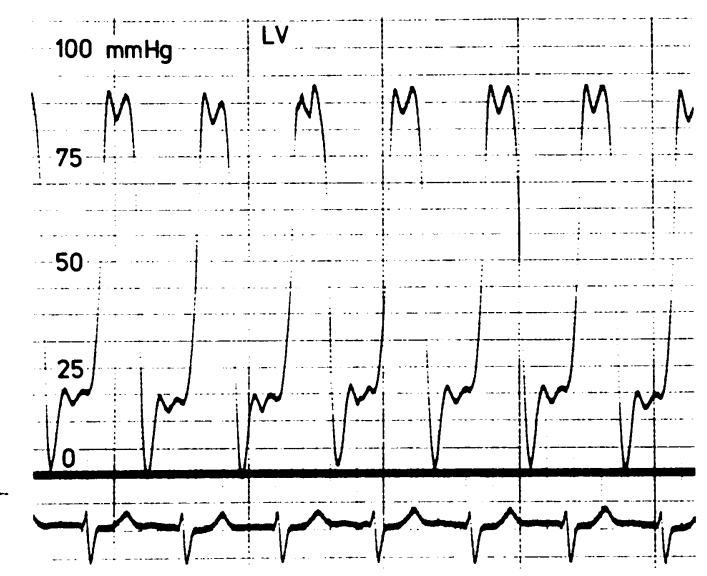

With time similar cases presented among relatives. A detailed family history suggested an hereditary cardiac disease and we examined four generations of the family (fig 1). Members of a fifth generation (cases I.1 and I.2) are also described but were not examined. Cases III.9 and III.10 are described in detail. The table shows data on 15 confirmed or probable cases out of 48 family members in the pedigree.

Those who were examined and thought to be unaffected were symptom free individuals with normal physical examination and electrocardiography. One unaffected individual had a normal echocardiogram and skeletal muscle biopsy (case IV.1).

\section{Case III.9}

In 1974 a forty year old woman was referred for emergency cardiac pacing because of atrioventricular block. The electrocardiogram showed 2:1 second degree atrioventricular block and left atrial enlargement, which was confirmed by echocardiography. This also showed that ventricular systolic function was good. Cardiac catheterisation showed normal coronary arteries and normal ventricular size and function. Pressure traces were not available for review. Endomyocardial biopsy specimens were taken. These were examined by light microscopy only and showed regularly arranged myocardial fibres with mild hypertrophy. There was no endomyocardial fibrosis. Congo red staining for amyloid was negative.

After pacing she remained well for eight years but then became progressively disabled by a non-wasting myopathy affecting principally the proximal and distal muscles of her arms and legs, and also by exertional dyspnoea. Electromyography confirmed a primary muscle disorder without evidence of myotonia. Skeletal muscle obtained from the pectoralis major when the pacemaker pulsegenerator was renewed showed some scanty fibrosis but no other histological abnormality. Biochemical analysis of this specimen showed higher activity of mitochondrial cytochrome oxidase than control specimens from the quadriceps. This may simply reflect differences in muscle fibre type. The activities of cytochrome oxidase and succinate cytochrome $c$ reductase were normal, as was oxidation of pyruvate and palmitoyl carnitine. There was no evidence of carnitine deficiency or amyloid infiltration. She died suddenly in July 1988. Pacemaker function was normal.

\section{Case III.10}

In 1980 a forty one year old man was referred with dyspnoea and chest pain. The electrocardiogram showed right bundle branch block, left axis deviation, and first degree heart block with right atrial enlargement. There were nonspecific abnormalities of the ST segment and T wave. Echocardiography showed global impairment of left and right ventricular systolic function with right ventricular hypertrophy and paradoxical septal motion. The right ventricle was enlarged but the left was of normal dimensions with impaired contraction. There was enlargement of both atria. At cardiac catheterisation contrast ventriculography showed poor systolic function of both ventricles. The coronary arteries were normal. All intracardiac pressures were raised and the left ventricular diastolic pressure showed the "dip and plateau" appearance often seen in myocardial restriction (fig 2). Endomyocardial biopsy specimens were taken from the left and right ventricles. These were examined under light microscopy only and showed no evidence of endomyocardial fibrosis. Congo red staining for deposition of amyloid was negative. Electrophysical studies showed normal atrioventricular conduction but in view of the family history permanent ventricular pacing was started.

In early 1987 a proximal left leg weakness developed and a biopsy specimen was taken of the quadriceps muscle. This showed some mild atrophy and fibrotic changes. A few abnormal fibres with dark staining edges were seen in trichrome and $\mathrm{NADH}$ stains. One vacuolated fibre was invaded by macrophages and contained fat globules. The activities of cytochrome oxidase and succinate cytochrome $c$ reductase were normal as was oxidation of pyruvate and palmitoyl carnitine. Carnitine concentrations were normal.

He died suddenly in 1987. Necropsy showed some dilatation of all cardiac chambers but principally of the right atrium and ventricle. There was extensive patchy fibrosis in the epicardium, myocardium, and endocardium. The atrioventricular valves were not affected and endomyocardial fibrosis was not seen. The His bundle was extensively fibrosed. There was mild coronary atherosclerosis. Pacemaker function was normal.

\section{Discussion}

We found that symptoms associated with this restrictive cardiomyopathy usually developed in the third or fourth decade, though children might be affected. Asymptomatic ventricular restriction may occur before pulmonary venous hypertension leads to symptoms or before atrioventricular block intervenes. Skeletal muscle weakness may occur some years after the initial cardiac manifestations, usually in the fifth decade. Asymptomatic impairment of ventricular diastolic function with raised dia- 
Clinical features and laboratory data in 15 individuals with restrictive cardiomyopathy

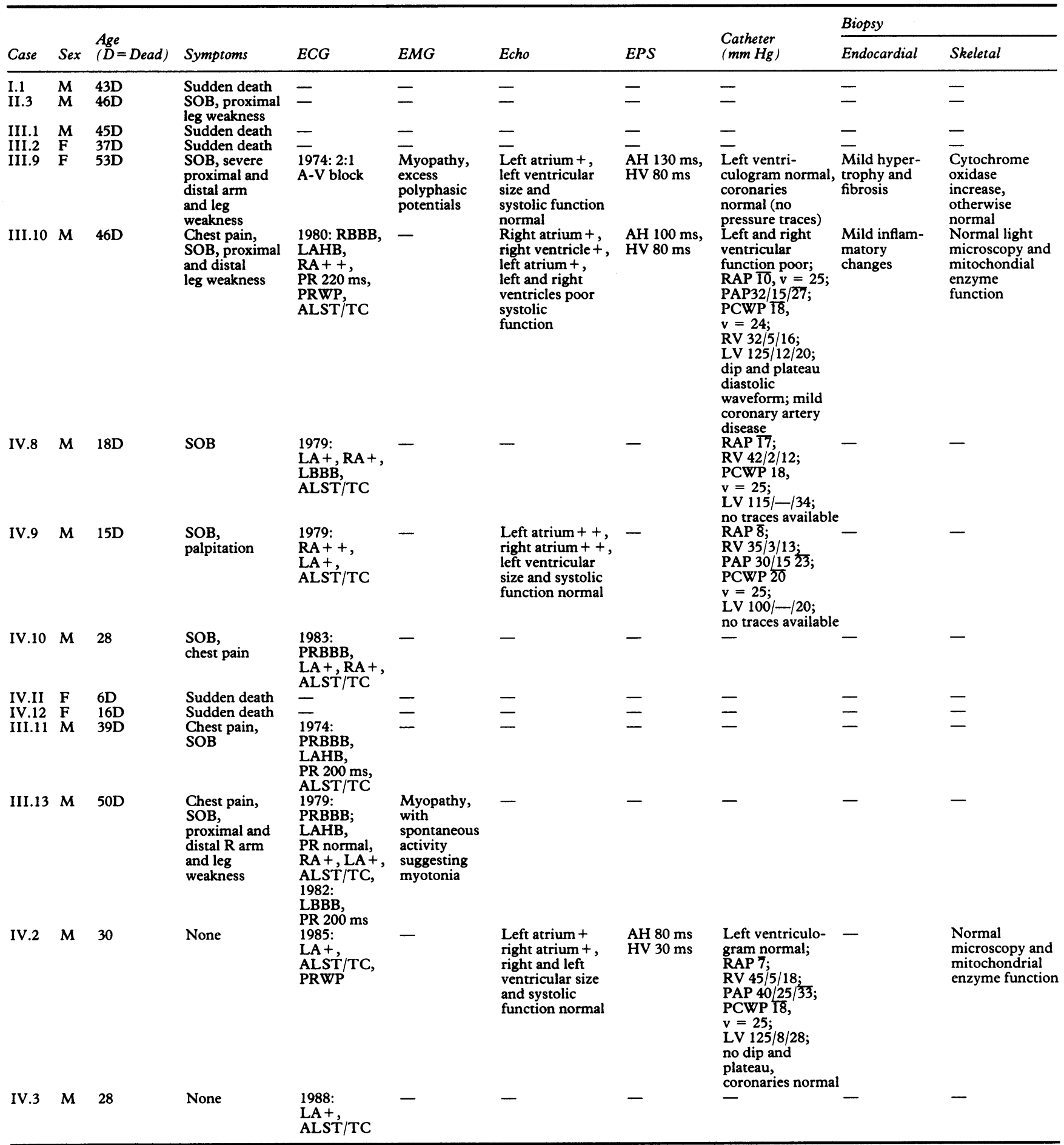

AH, conduction time from atrioventricular node to His bundle at intracardiac electrophysiological study; A-V, atrioventricular; ALST/TC, anterolateral ST segment and $T$ wave changes on surface electrocardiogram; ECG, surface electrocardiogram; Echo, cross sectional and $M$ mode echocardiography;

EMG, electromyogram; EPS, intracardiac electrophysiological study; HV, conduction time from His bundle to ventricle; LA +, left atrial enlargement on surface electrocardiogram by voltage criteria (+ + very enlarged); LAHB; left anterior hemifascicular block; LBBB, left bundle branch block; LV, left ventricle; No traces available, pressure traces at cardiac catheterisation not available for analysis (all catheter pressures are in mm $\mathrm{Hg}$ as indicated); PAP, pulmonary artery pressures; PCWP, pulmonary capillary wedge pressures; PRWP, poor $R$ wave progression in precordial leads; $R A$, right atrium; $R A P$, mean right atrial pressure; $R B B B$, right bundle branch block (PRBBB, partial block); RV, right ventricle; SOB, short of breath on exertion; $v=$ height of $v$ wave in PCWP.

stolic pressures may be the earliest manifestation of the disease. This was seen in case IV.2. In cases III. 9 and III.10 symptoms of cardiac failure persisted for many years after initial presentation without evidence of ventricular dilatation.

Two cases occurred in childhood (IV.8 and IV.9) and two more children who died suddenly are thought to have had the disease (cases IV.11 and IV.12). In cases IV.8 and IV.9 the disease was rapidly progressive leading to death in the second decade despite permanent pacing. Ventricular dimensions were normal although in cases III.10, IV.2, IV.8, and IV.9 both left and right ventricular diastolic pressures were raised (table 1). Left ventricular systolic function may be preserved after symptoms have developed, as in case III.9. The extent and location of fibrosis may determine the clinical course-for example the development of atrioventricular block or pulmonary venous hypertension caused by raised left ventricular 
Figure 3 An electrocardiogram of case III.13 from 1979 showing incomplete right bundle branch block, left axis deviation, left and right atrial enlargement, and $S T$ and $T$ wave abnormalities.
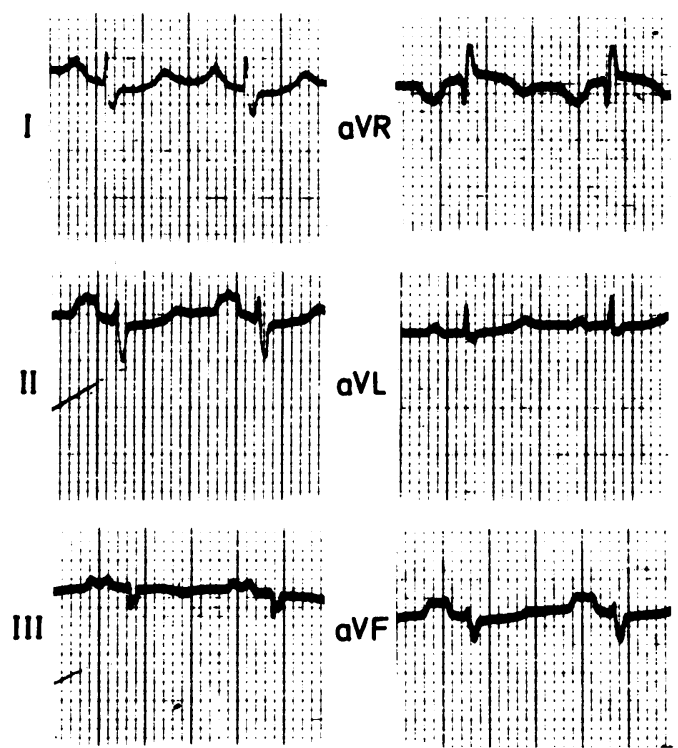

diastolic pressure. Poor left ventricular systolic function develops late in the course of the disease as does the skeletal myopathy. Left ventricular dilatation is not an early feature, perhaps because fibrosis stiffens the myocardium.

The electrocardiographic features were striking. Typically, $\mathrm{P}$ waves were exceptionally tall in the right precordial leads and broad in the standard leads owing to considerable enlargement of both atria (fig 3); this was confirmed by echocardiography (fig 4). First degree atrioventricular block was often seen. There were often partial or complete bundle branch block patterns; and attenuation of precordial $\mathrm{R}$ wave progression, ST segment changes, and $T$ wave abnormalities were common. Fibrosis in the conducting system accounts for the development of atrioventricular block. Electrophysiological studies were performed in three cases. In case III.9 there was clear evidence of prolongation of conduction times between the atrioventricular node and His bundle and the His bundle and ventricle. In case III.10 conduction times were normal but follow up after pacing showed that complete atrioventricular block had supervened. In the symptom free (case IV.2) electrophysiological studies were normal. The

Figure 4 Cross sectional echocardiogram from an apical four chamber view showing biatrial enlargement (the right ventricle and atrium lie on the right in frame).

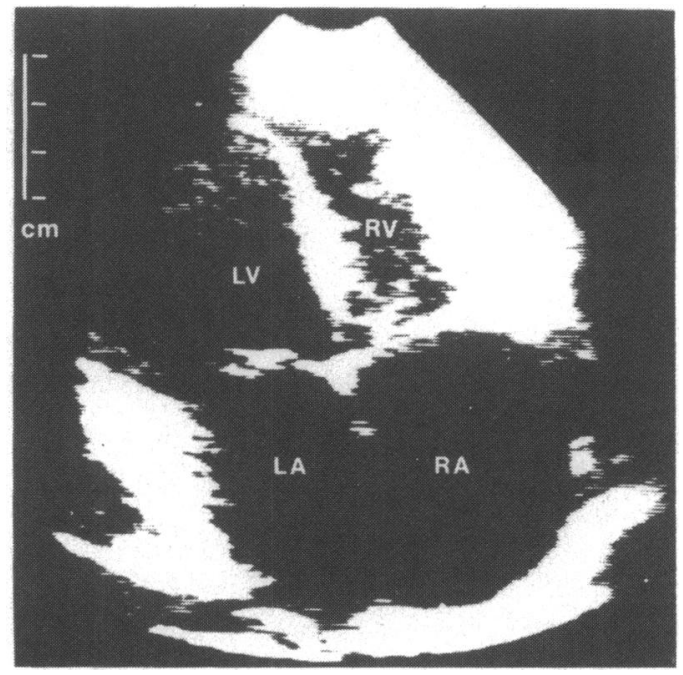

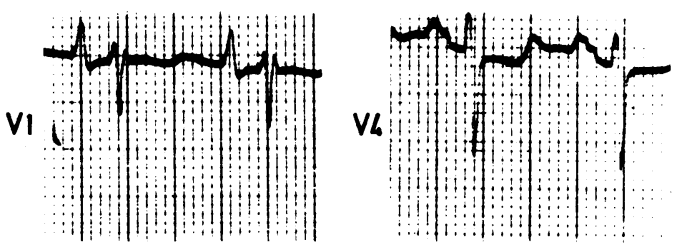
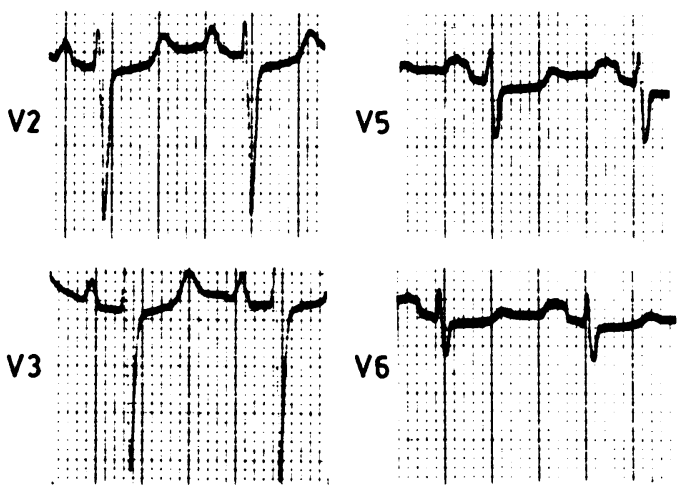

progress of disease in the conduction tissue was shown by changes in the surface electrocardiogram in case III.13. In 1979 the electrocardiogram showed partial right bundle branch block and left posterior fascicular block with a normal PR interval. Three years later there was complete left bundle branch block and first degree heart block.

Extensive patchy fibrosis was found throughout the heart in case III.10. Gross endocardial fibrosis is the pattern more typically associated with endomyocardial fibrosis and eosinophilic heart disease. Loffler's endomyocarditis, with inflammation, eosinophilic infiltration, and necrosis may be the acute form of such eosinophilic heart disease. ${ }^{1}$ Eosinophilia may be present at some stage in all cases of this type of restrictive cardiomyopathy, and D'Arbela et al recorded a mean survival of 24 months after presentation in one series. ${ }^{3}$ In eosinophilic heart disease, because the fibrotic process is mainly confined to the endocardium, atrioventricular block is not a feature and skeletal muscle involvement has not been described. Eosinophilia was not found at any stage in any of this family and the disease clearly has an insidious course in most cases.

The pathogenesis of this condition is unknown. Endocardial biopsy specimens obtained from two patients at the time of initial investigation were subjected to only very limited histological examination. At that time the possibility of a familial cardiomyopathy had not been identified. Carnitine deficiency, which has been associated with familial cardiomyopathy, ${ }^{4}$ was excluded in skeletal muscle specimens. Amyloid infiltration can produce impaired compliance ${ }^{56}$ by stiffening the myocardium, but was not seen in any specimen. Other specific heart muscle disorders that have been associated with restrictive haemodynamic function are haemochromatosis ${ }^{7}$ and collagen diseases such as scleroderma, ${ }^{8}$ but there was no evidence of these diseases in the family we studied.

There are reports of similar cases to those in this family. Benotti et al described nine patients 
(from a retrospective analysis of cardiac catheterisation data) with restrictive ventricular diastolic pressure profiles and reported a good outcome. ${ }^{9}$ Two endocardial biopsies were performed and in both the specimens were normal. Seigel et $a l^{10}$ reported four cases of idiopathic restrictive cardiomyopathy with many similar features to this family. The disease was characterised by restrictive haemodynamic dysfunction, atrial dilatation, and, in one patient, the development of atrioventricular block. In this series the mean time of survival was nine years after cardiac symptoms had developed. Necropsies were performed in all four patients. There was no endomyocardial fibrosis typical of eosinophilic heart disease. ${ }^{11}$ There was considerable dilatation of both atria and patchy endocardial fibrosis, with appreciable fibrotic infiltration of the myocardium that spared the atrioventricular valves. The patient with atrioventricular block had extensive $\mathrm{His}$ bundle fibrosis. None of these patients, however, is reported to have been related, nor does skeletal muscle weakness feature in these reports.

We report a familial disease of heart muscle producing myocardial restriction, apparently inherited as an autosomal dominant, and associated with atrioventricular block and a non-wasting skeletal myopathy. Heart block results from fibrosis in the conducting system. No endomyocardial fibrosis was seen, but fibrosis was found throughout the myocardium and epicardium at necropsy. This may be the end result of the pathological process and the cause of restriction.

We thank Dr Rocco Macri of the Divisione di Cardio-Chirurgia Infantile, Presidio Ospedaliero di Massa, Massa Carrera, Italy, for his help in providing clinical material. We also thank $\mathrm{Dr}$ Emanuel for advice, Dr E G J Olsen for the histopathological interpretation of the endocardial biopsy specimens and the necropsy specimen, and Dr Joan Round of University College London, for obtaining and examining the biopsy specimens of skeletal muscle. This study was supported by the Nuffield Foundation.

1 Oakley CM, Olsen EGJ. Eosinophilia and heart disease. Br Heart J 1977;39:233-7.

2 Olsen EGJ. The pathology of the cardiomyopathies. A critical analysis. Am Heart J 1979;98:385-92.

3 D'Arbela PG, Mutazindwa T, Patel AK, Somers K. Survival after first presentation with endomyocardia fibrosis. Br Heart J 1972;34:403-7.

4 Waber LJ, Valle D, Neill C, DiMarco S, Shug A. Carnitine deficiency presenting as familial cardiomyopathy: a treatable defect in carnitine transport. $J$ Pediatr 1982;101 $700-4$.

5 Swanton RH, Brooksby IAB, Davies MJ, Coltart DJ, Jenkins BS, Webb-Peploe MM. Systolic and diastolic ventricular function in cardiac amyloidosis. $A m J$ Cardiol 1977;39:658-63.

6 Buja LM, Khoi NB, Roberts WC. Clinically significan cardiac amyloidosis: clinicopathological findings in cardiac amyloidosis: clinicopathologica

7 Cutler DJ, Isner JM, Bracey AW, et al. Hemochromatosis heart disease: an unemphasized cause of potentially reversible restrictive cardiomyopathy. $\mathrm{Am} \mathrm{J}$ Med 1980 69:923-6.

8 Seigel RJ, O'Connor B, Ismael M, Criley JM. Left ventricular function in scleroderma [Abstract]. Circulation 1980;62:1228.

9 Benotti JR, Grossman W, Cohn PF. Clinical profile of restrictive cardiomyopathy. Circulation 1980;61: 1206-12.

10 Seigel RJ, Prediman KS, Fishbein MC. Idiopathic restrictive cardiomyopathy. Circulation 1984;70:165-9.

11 Report of the WHO/ISFC task force on the definition and classification of cardiomyopathies. Br Heart J 1980; 44:672-3. 